\title{
NÍVEL DE INTENSIDADE NAS AULAS DE EDUCAÇÃO FÍSICA DO ENSINO FUNDAMENTAL
}

\author{
Fernando Vian \\ Universidade Federal do Rio Grande do Sul, Porto Alegre, Rio Grande do Sul, Brasil. \\ Augusto Pedretti \\ Universidade Federal do Rio Grande do Sul, Porto Alegre, Rio Grande do Sul, Brasil. \\ Júlio Brugnara Mello \\ Universidade Federal do Rio Grande do Sul, Porto Alegre, Rio Grande do Sul, Brasil. \\ Naildo Santos Silva \\ Universidade Federal do Rio Grande do Sul, Porto Alegre, Rio Grande do Sul, Brasil. \\ Luciano Panosso da Silva \\ Universidade Regional Integrada do Alto Uruguai e das Missões, Erechim, Rio grande do Sul, Brasil. \\ Adroaldo Cezar Araujo Gaya \\ Universidade Federal do Rio Grande do Sul, Porto Alegre, Rio Grande do Sul, Brasil.
}

\begin{abstract}
Resumo
O objetivo deste estudo foi descrever os níveis de intensidade das aulas de Educação Física de escolares do ensino fundamental. Trata-se de uma pesquisa exploratória com abordagem quantitativa e corte transversal. A escola e a turma observada foram selecionadas por conveniência, devido ao vínculo de estágio anterior à pesquisa. Foram avaliadas 11 aulas, um único aluno (13-14 anos) por aula. As variáveis analisadas durante a aula de Educação Física foram: as atividades em que os escolares estavam envolvidos, a frequência cardíaca, a velocidade e a distância percorrida. Para o tratamento dos dados, foram utilizadas técnicas descritivas. Apenas 31 minutos foram aproveitados do período total da aula, no qual permaneceram 19 minutos em intensidade muito baixa e baixa. Sendo assim, os alunos avaliados apresentaram um baixo aproveitamento no tempo de aula, ademais, os esforços físicos realizados com intensidade alta caracterizaram-se como de curta duração.
\end{abstract}

Palavras-chave: Educação Física. Esforço físico. Ensino fundamental e médio.

\section{INTENSITY LEVEL OF PHYSICAL EDUCATION CLASSES IN ELEMENTARY SCHOOL}

\begin{abstract}
The aim of this study was describe the intensity levels of Physical Education classes of elementary schoolchildren. This is an exploratory research with a quantitative and crosssectional approach. The school and class observed were selected for convenience because of the previous stage link to the survey. We evaluated 11 classes, one student (13-14 years) per class. The variables analyzed during the Physical Education class were: activities in which
\end{abstract}


schoolchildren were involved, heart rate, speed and distance traveled. Descriptive techniques were used for the treatment of the data. Only 31 minutes were taken of the total period of the class in which they remained 19 minutes in very low and low intensity. Thus, the evaluated students presented a low achievement in the class time, in addition the physical efforts realized with high intensity were characterized as of short duration.

Keywords: Physical Education. Physical exertion. Education, Primary and Secondary.

\section{NIVEL DE INTENSIDAD EN LAS CLASES DE EDUCACIÓN FÍSICA DE LA EDUCACIÓN PRIMARIA}

\section{Resumen}

El objetivo de este estudio fue describir los niveles de intensidad de las clases de Educación Física de alumnos de la educación primaria. Se trata de una investigación exploratoria con enfoque cuantitativo y corte transversal. La escuela y una clase del octavo año fueron seleccionadas debido al vínculo de pasantía anterior a la investigación. Se evaluaron 11 clases, un alumno (13-14 años) por clase. Las variables analizadas durante la clase de Educación Física fueron: las actividades en las que los alumnos estaban involucrados, la frecuencia cardíaca, la velocidad y la distancia recorrida. Para el tratamiento de los datos se utilizaron técnicas descriptivas. Sólo 31 minutos del total de la clase fueron aprovechados, durante esos minutoslos alumnos permanecieron 19 minutos en intensidad muy baja y baja. Por lo tanto, los alumnos evaluados presentaron un bajo aprovechamiento en el tiempo de clase. Además de eso, los esfuerzos físicos realizados con intensidad alta se caracterizaron como de corta duración.

Palabras clave: Educación Física. Esfuerzo físico. Educación Primaria y Secundaria.

\section{Introdução}

A Organização Mundial da Saúde (WHO, 2010) recomenda que crianças e adolescentes de 5 a 17 anos devem acumular pelo menos 60 minutos de atividade física (AF) com intensidade moderada a vigorosa diariamente. Algumas evidências apontam que a prática regular de AF é um dos principais comportamentos associados à prevenção da saúde em geral, principalmente em crianças (STRONG et al., 2005; JANSSEN; LEBLANC, 2010; SALLIS et al., 2016).

Não obstante, o nível de AF de crianças em todo o mundo tem diminuído substancialmente (HALLAL et al., 2012; SALLIS et al., 2016). Nessa perspectiva, diversas intervenções têm sido propostas com o objetivo de minimizar esse decréscimo supracitado, assim como o aumento do comportamento sedentário. Dentre essas estratégias, encontram-se os programas de intervenção na Educação Física escolar (EFesc). Uma série de intervenções cujo objetivo é a promoção da $\mathrm{AF}$ na perspectiva da saúde tem demonstrado efeitos favoráveis em diversos indicadores: aptidão física, saúde mental, cognição, massa óssea e saúde cardiovascular (KAHN et al., 2002; MCKAY et al., 2000; MEYER et al., 2014; OLIVEIRA et al., 2017).

A principal característica das intervenções que apontam ganhos em parâmetros de saúde é a inserção de uma parte da aula com atividades com intensidade moderada a vigorosa (DOBBINS et al., 2013). Entretanto, indo de encontro a essas propostas, alguns estudos evidenciaram que as aulas de EFesc, no Brasil, não apresentam como característica a prática de AF de forma a impactar positivamente em ganhos ou na manutenção dos indicadores de 
saúde (GUEDES; GUEDES, 2001; KREMER; REICHERT; HALLAL, 2012; HINO et al., 2012; SANTOS et al., 2017).

Com relação às aulas de EFesc no ensino fundamental, as evidências sobre as características das aulas e a intensidade das atividades durante as aulas são escassas. Kremer, Reichert e Hallal (2012) objetivaram avaliar a intensidade e a duração dos esforços físicos durante as aulas de EFesc no ensino fundamental e médio. Neste estudo, os autores observaram 218 aulas e avaliaram, através de acelerometria, 272 estudantes em Pelotas - RS. Os resultados indicaram que o tempo médio de duração das aulas foi de 35 minutos, dos quais $33 \%$ estavam em AF moderada a vigorosa.

A abordagem dos programas escolares sobre a $\mathrm{AF}$ e a sua relação com a saúde são pouco conhecidas no Brasil. Portanto, pouco se sabe se a EFesc no ensino fundamental tem a preocupação com a promoção de AF com intensidade, duração e frequência suficientes para que ocorram benefícios para a saúde. Nesse sentido, estudos que evidenciem as características de intensidade e atividades praticadas nas aulas de EFesc podem indicar, mesmo que minimamente, o que tem sido realizado nas escolas. Assim sendo, justificamos nosso estudo, que tem como objetivo descrever os níveis de intensidade das aulas de Educação Física de escolares do ensino fundamental de uma escola do município da microrregião do Médio Alto Uruguai.

\section{Procedimentos metodológicos}

Trata-se de uma pesquisa de cunho exploratório com abordagem quantitativa e corte transversal (GAYA et al., 2016).

\section{Estudo exploratório}

A pesquisa foi realizada em uma escola pública da rede estadual de ensino do estado do Rio Grande do Sul, na cidade de Frederico Westphalen, localizada na região noroeste do estado. A estrutura escolar do ensino fundamental da cidade é composta por 13 escolas estaduais e nove escolas municipais. Sua estrutura conta com 19 salas de aula, laboratório de informática e de ciências, biblioteca, auditório, refeitório, ginásio poliesportivo, entre outros espaços para o desenvolvimento dos alunos. A disciplina de EFesc é oferecida aos anos iniciais e finais do ensino fundamental, ao ensino médio e à educação de jovens e adultos em dois períodos de 45 minutos semanais.

A escola-caso foi selecionada por conveniência, pelo fato de que um dos pesquisadores tinha vínculo de estágio anterior à pesquisa. A escola conta com 215 adolescentes matriculados de forma regular nos anos finais do ensino fundamental e três professores de Educação Física. O professor responsável pelos anos finais do ensino fundamental foi convidado a participar do estudo e, após o aceite, por sorteio, apenas uma das suas turmas foi selecionada. A logística da pesquisa e a disponibilidade de materiais não permitiram que mais turmas fossem avaliadas.

A turma sorteada foi o $8^{\circ}$ ano do turno da tarde, a qual foi informada sobre os propósitos de pesquisa, com a solicitação da autorização (assinatura) dos pais ou responsáveis, através do termo de consentimento livre e esclarecido, e também do consentimento individual de cada adolescente. O estudo foi aprovado pelo Comitê de Ética em Pesquisa em Seres Humanos da Universidade Regional Integrada do Alto Uruguai e das Missões, sob o parecer número 48984615.8.0000.5352. 


\section{Variáveis e procedimentos de coleta dos dados}

Uma aula semanal foi avaliada em um período de três meses. Em um momento prévio à aula, um aluno era sorteado a partir da lista completa de nomes da turma. Caso o aluno não estivesse presente, o sorteio era realizado novamente. Portanto, foi avaliado um aluno em cada aula, perfazendo um total de 11 aulas.

As variáveis que compuseram o estudo foram o tipo de atividade em que os escolares estavam envolvidos, a frequência cardíaca (FC) e a velocidade e a distância total percorrida nas aulas de EFesc.

Para verificar o tipo de atividade em que o escolar estava envolvido, foi utilizado o instrumento adaptado de Guedes e Guedes (2001). Essa adaptação foi realizada, pois a pesquisa original do instrumento analisou aulas de 50 minutos e nós analisamos aulas de 45 minutos, ou seja, as aulas observadas foram divididas em 540 períodos de cinco segundos. Para descrever o tipo de atividade, foram utilizadas as categorias propostas pelo instrumento.

A FC foi avaliada através de um dispositivo cardíaco portátil (Garmin, modelo Forerunner 310XT). Esse aparelho registra com precisão, por meio de dados de GPS, a posição, a distância, o ritmo, a frequência cardíaca $(2,4 \mathrm{~Hz})$ e as medidas de gasto energético. O Forerunner 310XT monitora continuamente os batimentos por minuto juntamente dos dados previamente inseridos no monitor para mensurar as calorias. A transferência dos dados é feita via wi-fi (sem fio) para o software Garmin Connect, para análise e compartilhamento. O sensor (cinta) para monitorar a frequência cardíaca foi fixado na altura do tórax do adolescente e um dispositivo GPS (relógio propriamente dito) foi utilizado no punho. Previamente ao início das aulas de EFesc, o sensor e o relógio eram colocados no aluno sorteado, e os indicadores foram registrados continuamente durante os 45 minutos de aula. Para a classificação da intensidade, foi considerada que uma FC $<119$ batimentos por minuto (bpm) era de intensidade muito baixa, uma FC entre 120-149 de intensidade baixa, entre 150169 de intensidade moderada e uma FC >170 de intensidade vigorosa (GUEDES; GUEDES 2001). Através do dispositivo cardíaco portátil (Garmin, modelo Forerunner 310XT), a velocidade foi classificada em: parado - $0 \mathrm{~km} / \mathrm{h}$; caminhada leve - de 0,1 a $3 \mathrm{~km} / \mathrm{h}$; caminhada moderada - 3,1 a $6 \mathrm{~km} / \mathrm{h}$; trote $-6,1$ a $9 \mathrm{~km} / \mathrm{h}$; corrida - acima de $9 \mathrm{~km} / \mathrm{h}$. A distância foi avaliada através do dispositivo cardíaco portátil (Garmin, modelo Forerunner 310XT) e dividida em <500, 501-1000, >1001 metros percorridos durante a aula de EFesc.

\section{Procedimentos estatísticos}

Para o tratamento dos dados, foram utilizadas técnicas de análises descritivas. Para as variáveis de tempo, primeiramente foi calculado o tempo de cada aluno (utilizando frequências relativas), logo após foi calculado o tempo médio. A distância foi descrita de forma individual e foram calculados a média e o desvio-padrão de todos os alunos.

\section{Resultados}

A amostra foi constituída por 11 adolescentes dos anos finais do ensino fundamental (sete meninos e quatro meninas), com idades entre 13 e 14 anos.

No Gráfico 1, foi apresentada a frequência do tipo de atividade em que os escolares estavam envolvidos durante as aulas de EFesc (média das 11 aulas observadas de um único aluno por aula). Os resultados nos mostram que aproximadamente 31 minutos foram verdadeiramente aproveitados do período total da aula, uma vez que os 14 minutos restantes foram gastos para o deslocamento de início e término da aula e também para se vestirem adequadamente para a aula de EFesc. A partir desse tempo de aula aproveitada, foi observada 
a frequência do tipo de atividade em que os escolares estavam envolvidos (um único aluno por aula).

As subdivisões das aulas tiveram uma distribuição de tempo muito desparelha. Individualmente, os alunos permaneceram mais tempo em momentos de jogos propriamente ditos e administração/organização de tarefas.

Dos 31 minutos de aula efetivamente aproveitados (média das 11 aulas observadas de um único aluno por aula), um pouco mais de um quarto da aula foi despendido parado e/ou em caminhada leve, portanto, aproximadamente 23 minutos da aula foram realmente em intensidade moderada ou vigorosa.

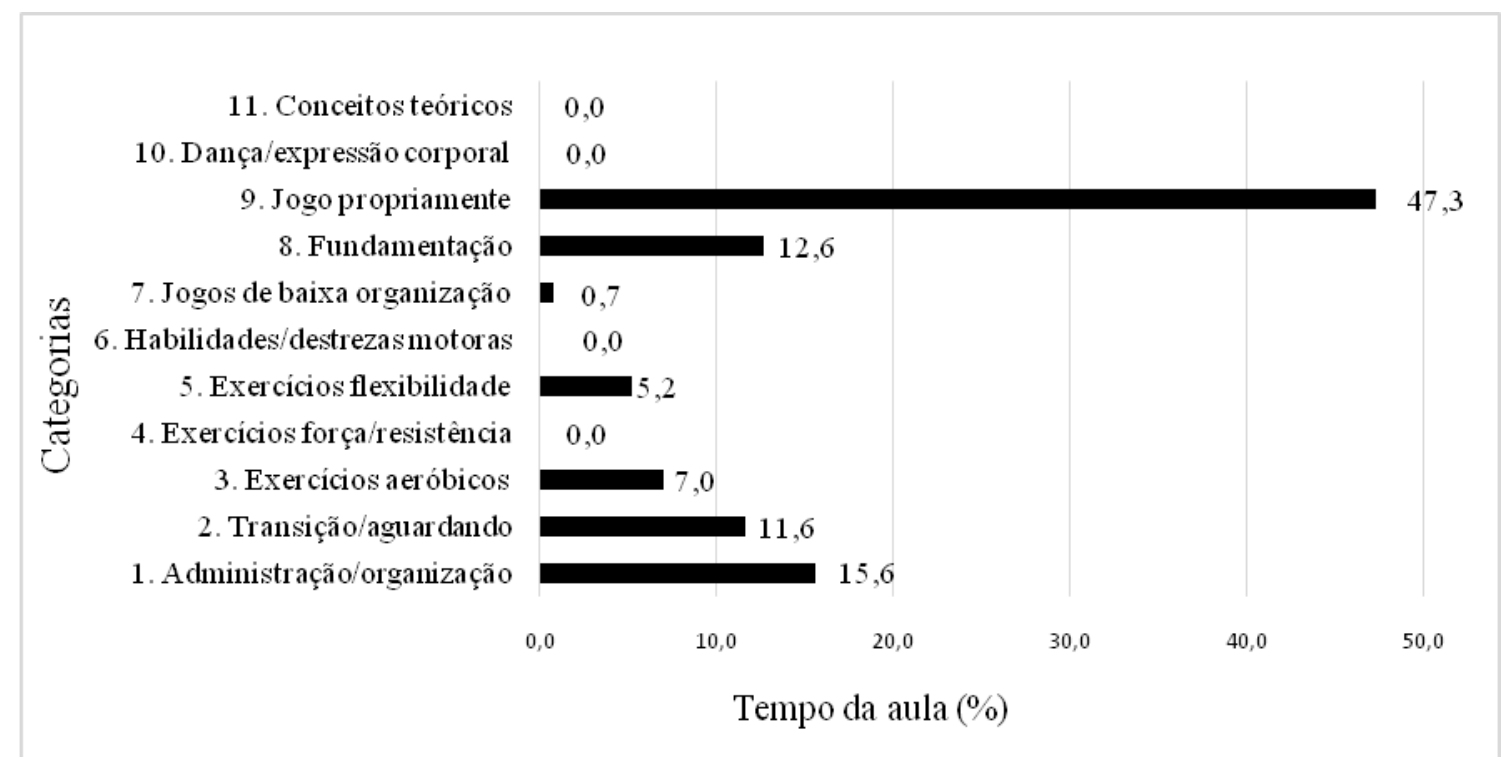

Gráfico 1. Frequência média do tipo de atividade em que os escolares estavam envolvidos durante as aulas de Educação Física.

Fonte: Elaborado pelos autores, 2018.

O Gráfico 2 apresenta o tempo despendido pelos alunos (um aluno por aula) da FC média durante as aulas de EFesc. Sendo considerado o tempo total aproveitado de 31 minutos, podemos observar que os alunos permaneceram mais da metade do tempo em intensidade muito baixa ou baixa (nove e dez minutos, respectivamente). Despenderam cinco minutos em atividades de intensidade moderada e nove minutos em atividades de intensidade vigorosas. 


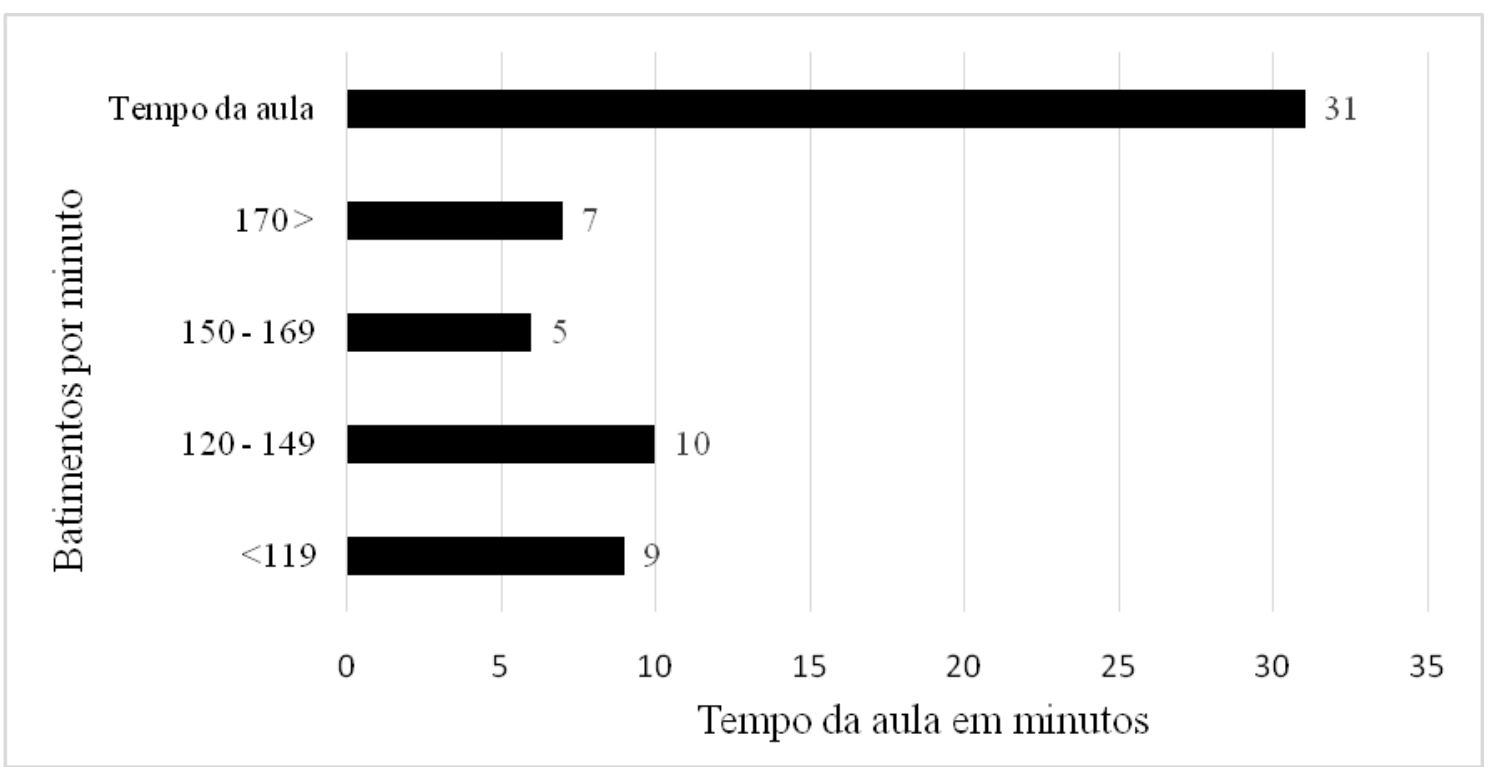

Gráfico 2. Frequência cardíaca média dos alunos durante as aulas de Educação Física. Fonte: Elaborado pelos autores, 2018.

A velocidade despendida durante as aulas de EFesc está apresentada no Gráfico 3. Podemos observar que, na maior parte da aula, um aluno por aula, eles permaneciam parados ou em caminhada (6 e 20 minutos, respectivamente), e somente cinco minutos em trote ou corrida.

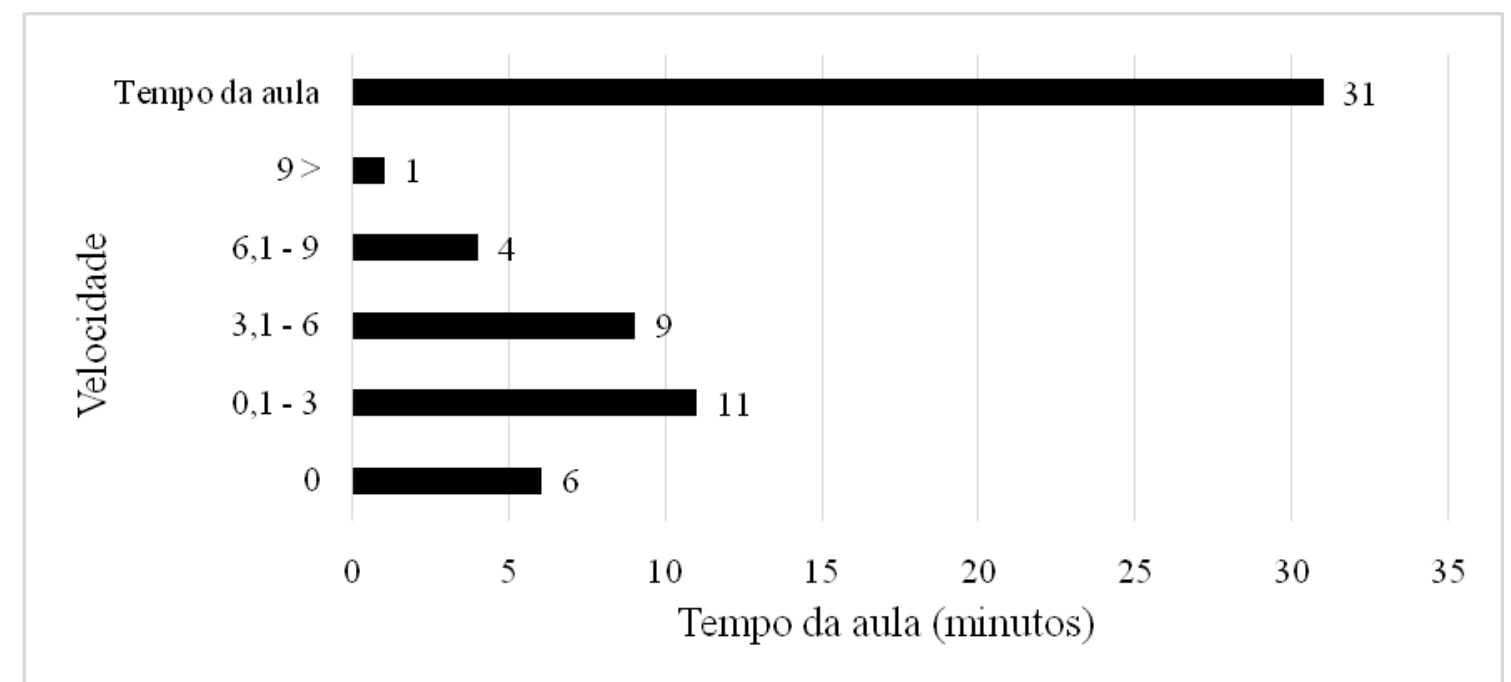

Gráfico 3. Velocidade média dos alunos durante as aulas de Educação Física.

Fonte: Elaborado pelos autores, 2018.

O Gráfico 4 apresenta informações quanto à distância percorrida pelos alunos, um aluno por aula, durante as aulas. Nota-se que a contagem das aulas avaliadas tem um somatório de dez aulas, ocorrendo, portanto, a perda de uma aula, pois, no dia de avaliação, as condições climáticas (chuva) impossibilitaram o bom funcionamento do dispositivo utilizado. Tivemos quatro aulas em que os alunos percorreram uma distância superior a 1001 metros, outras quatro aulas tiveram uma distância percorrida entre 501-1000 metros, e duas aulas nas quais os alunos percorreram uma distância menor que 500 metros. 


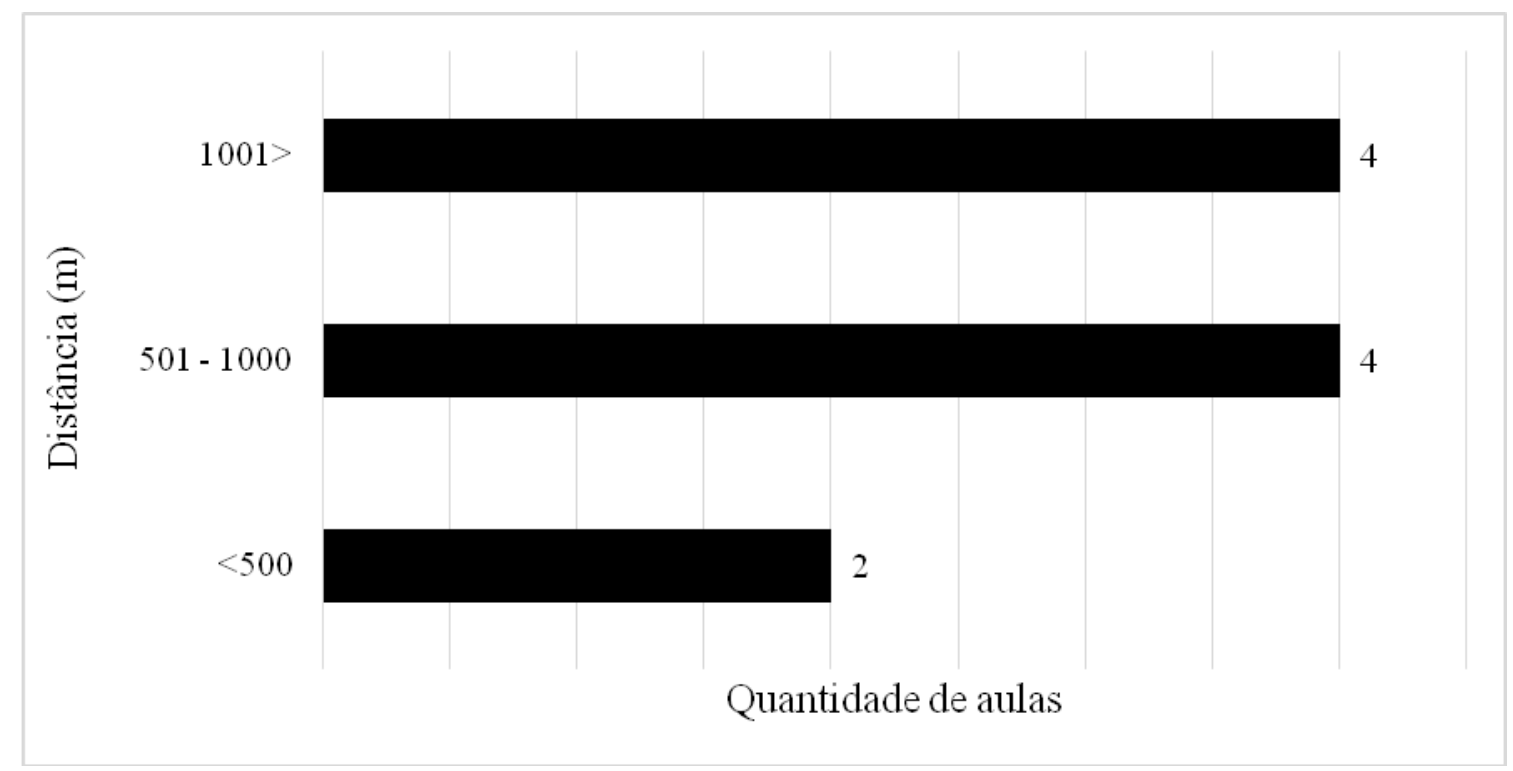

Figura 4. Distância percorrida pelo aluno durante a aula de Educação Física.

Fonte: Elaborada pelos autores, 2018.

\section{Discussão}

Este estudo se propôs a descrever o tipo de atividade em que os escolares estavam envolvidos nas aulas de EFesc, a intensidade através da FC, a velocidade e a distância total percorrida em escolares do $8^{\circ}$ ano de uma escola pública da rede estadual de ensino do RS. Visto que a EFesc se caracteriza como uma disciplina singular, sendo a única a promover diretamente a saúde e diversas linguagens do movimento humano, através do ensino de um estilo de vida ativo e saudável, a prática pedagógica adotada pelo professor nas aulas de EFesc reflete diretamente sobre o comportamento, a atitude e, consideravelmente, no nível de aprendizado dos alunos.

O período de duração médio das aulas de EFesc (31 minutos) indica que as aulas não são aproveitadas por completo. Um tempo médio semelhante na duração das aulas de EFesc foi encontrado por outros pesquisadores no Brasil. Kremer, Reichert e Hallal (2012) apontam que as aulas de EFesc na cidade de Pelotas - RS tiveram 35 minutos de aproveitamento quando analisados os adolescentes da rede municipal de educação. Guedes e Guedes (2001), em estudo realizado com adolescentes da rede estadual de ensino da cidade de Londrina - PR, evidenciaram tempo médio de 37 minutos (74\%) de um total de 50 minutos disponibilizados, ao encontro do evidenciado nos EUA (NADER, 2003). Portanto, nossos resultados corroboram o apresentado na literatura, na qual se identifica que a troca de uniforme e o deslocamento dos estudantes para a aula de EFesc são fatores importantes a serem considerados, pois diminuem de forma significativa o tempo disponível para a realização das atividades propostas.

Os resultados encontrados em nosso estudo quanto à prática do jogo propriamente dito (47\% dos 31 minutos aproveitados) se assemelha aos achados do estudo de Hino, Reis e Añes (2012), realizado junto a adolescentes de uma escola da rede pública de ensino da cidade de Curitiba - PR, em que os alunos permaneceram aproximadamente 53\% em atividades de jogos estruturados de um tempo total de 50 minutos de aula.

Hino, Reis e Añes (2012) também evidenciaram que, em média, os alunos permaneceram cerca de $9 \%$ em momentos de administração/organização e transição/aguardando para realizar as atividades. Evidenciamos que, somados, $27,2 \%$ das aulas observadas foram destinados a administração/organização e transição/aguardando, respectivamente, e que, se somados aos 12,6\% utilizados para fundamentação, teremos um 
total de aproximadamente $40 \%$ (dos 31 minutos aproveitados) de não prática. Entendemos que esses momentos são importantes para um bom andamento do plano de aula proposto, mas devem ser melhor aproveitados, como nos traz Almeida e Franco (2011), que recomendam que as aulas de EFesc tenham um tempo maior dedicado à prática (de AF) para promover efetivamente o ensino e a aprendizagem.

Diante dos benefícios à saúde física (PEREIRA et al., 2014; MOSER et al., 2011) e mental obtidos através da prática regular de AF (RIEK et al., 2013; HALLAL et al., 2006), pode-se apontar a aula de EFesc como de suma importância para que as crianças e adolescentes atinjam níveis recomendados de AF (GUEDES et al., 2007). Além do mais, a EFesc é apontada como um ambiente favorável para aumentar os níveis de AF em crianças e adolescentes, intervindo assim na promoção da saúde e aumentando também os níveis de esforços (SALLIS et. al, 2016).

Entre as aulas analisadas, identificou-se que os alunos permaneceram aproximadamente 13 minutos em atividades de intensidade moderada a vigorosa. Guedes e Guedes (2001) encontraram um tempo de 17 minutos, de uma aula de 50 minutos, em que os alunos estavam em atividade de intensidade moderada a vigorosa. Outros estudos (KREMER; REICHERT; HALLAN, 2012; NADER, 2003) mostraram resultados similares, com tempo médio de 12 minutos em atividade com intensidade moderada a vigorosa. Porém, as aulas avaliadas do estudo de Kremer, Reichert e Hallal (2012) tinham 45 minutos, já as aulas do estudo de Nadar (2003), 50 minutos.

Apesar do reconhecimento da aptidão física como padrão de condicionamento físico capaz de propiciar a prática de AF vigorosa individual, a qual é analisada sob aspectos relacionados à saúde e ao desempenho motor (BOUCHARD; SHEPARD; STEPHENS, 1994), observamos um baixo tempo despendido em atividades de intensidade moderada a vigorosa nas aulas de EFesc. A fim de que os professores possam dinamizar suas aulas em consonância com a Base Nacional Comum Curricular e dentro das competências específicas da Educação Física, é que surge a proposta de Oliveira et al. (2017).

Oliveira e colegas propuseram 15 minutos de formação corporal, ou seja, exercícios funcionais realizados com movimentos naturais como pular, correr, puxar, agachar, girar e empurrar, cujo objetivo foi desenvolver a força, o equilíbrio, a flexibilidade, a resistência, a velocidade e a agilidade na parte inicial da aula. Essa estratégia se mostrou efetiva na melhora dos níveis de aptidão física relacionados à saúde de 88 adolescentes em uma escola da rede privada de Porto Alegre - RS.

O tempo em que os alunos estavam na administração/organização e transição/aguardando influenciou diretamente nos seis minutos em que estiveram parados e nos 11 minutos em caminhada leve. A distância percorrida durante o período de aula variou dentre as aulas observadas. Em duas aulas, o aluno não chegou a percorrer 500 metros em 31 minutos de aula, enquanto em quatro aulas o aluno percorreu uma distância superior a 1001 metros. Essa discrepância nos resultados pode ser explicada pela metodologia e pelas atividades aplicadas e também por ter sido sorteado somente um único aluno da turma para avaliação.

Esses resultados nos mostram que os alunos se movimentam pouco durante as aulas de EFesc. A fim de que se pudesse classificar crianças e adolescentes quanto a indicadores de saúde cardiometabólica, o PROESP-Br (GAYA; GAYA, 2016) propôs pontos de cortes para a capacidade aeróbica avaliada através do teste de caminhada/corrida dos seis minutos. Os adolescentes de 13 a 14 anos devem percorrer aproximadamente 1000 metros para serem classificados dentro da zona saudável, porém, como percebemos em duas aulas, os alunos percorreram distâncias inferiores a 1000 metros em um tempo de 31 minutos.

Portanto, algumas hipóteses e sugestões foram originadas a partir dos resultados do presente estudo. $\mathrm{O}$ fato de os alunos avaliados apresentarem uma característica de 
envolvimento na aula muito semelhante nos permite indicar que possivelmente as aulas de Educação Física apresentam características parecidas, embora a generalização não seja o objetivo central deste estudo.

As aulas foram pouco aproveitadas, despendendo um longo período de tempo em atividades que não contemplam o aumento dos níveis de $\mathrm{AF}$, como permanência em categorias de aulas como administração/organização e transição/aguardando. Já os esforços físicos realizados caracterizaram-se como de curta duração, tendo assim pouco tempo de permanência em intensidade moderada e vigorosa. Os alunos permaneceram aproximadamente metade da aula parados ou em caminhada leve, acarretando as curtas distâncias que foram percorridas.

\section{Referências}

ALMEIDA, F. J.; FRANCO, M. G. Avaliação para aprendizagem: o processo avaliativo para melhorar o desempenho dos alunos. São Paulo: Ática Educadores, 2011.

BOUCHARD, C. E.; SHEPHARD, R. J.; STEPHENS, T. E. Physical activity, fitness, and health: international proceedings and consensus statement. In: INTERNATIONAL CONSENSUS SYMPOSIUM ON PHYSICAL ACTIVITY, FITNESS, AND HEALTH, 2nd, 1992. Annals... Toronto: Human Kinetics Publishers, 1994.

DARIDO, S. C. A educação física na escola e o processo de formação dos não praticantes de atividade física. Revista Brasileira de Educação Física e Esporte, v. 18, n. 1, p. 61-80, jan./mar. 2004.

DOBBINS, M. et al. School-based physical activity programs for promoting physical activity and fitness in children and adolescents aged 6-18. The Cochrane Library, v. 21, n. 1, 2013.

GAYA, A; GAYA AR. Manual de aplicação de medidas e testes, normas e critérios de avaliação. Projeto Esporte Brasil, Porto Alegre, 2016.

et al. Projetos de pesquisa científica e pedagógica: o desafio da iniciação científica. Belo Horizonte: Casa da Educação Física, 2016.

GUEDES, D. P.; GUEDES, J. E. R. P. Esforços físicos nos programas de educação física escolar. Revista Paulista de Educação Física, v. 15, n. 1, p. 33-44, 2001.

. et al. Daily energy expenditure and plasmatic lipid-lopoprotein levels in adolescents.

Revista Brasileira do Medicina do Esporte, v. 13, n. 2, p. 107-111, mar./abr. 2007.

HALLAL, P. C. et al. Adolescent physical activity and Health. The American Orthopaedic Sports Medicine Journal, Rosemont, v. 36, n. 12, p. 1019-1030, 2006.

. et al. Global physical activity levels: surveillance progress, pitfalls, and prospects.

The Lancet, v. 380, n. 9838, p. 247-257, 2012.

HINO, A. A. F.; REIS, R. S.; AÑEZ, C. R. R. Observação dos níveis de atividade física, contexto das aulas e comportamento do professor em aulas de educação física do ensino 
médio da rede pública. Revista Brasileira de Atividade Física \& Saúde, v. 12, n. 3, p. 21$30,2012$.

JANSSEN, I.; LEBLANC, A. G. Systematic review of the health benefits of physical activity and fitness in school-aged children and youth. International Journal of Behavioral Nutrition and Physical Activity, v. 7, n. 1, p. 40, 2010.

KAHN, E. B. et al. The effectiveness of interventions to increase physical activity. American Journal of Preventive Medicine, v. 22, n. 4, p. 73-107, 2002.

MEYER, U. et al. Long-term effect of a school-based physical activity program (KISS) on fitness and adiposity in children: a cluster-randomized controlled trial. PloS one, v. 9, n. 2, p. e87929, 2014.

MCKAY, H. A. et al. Augmented trochanteric bone mineral density after modified physical education classes: a randomized school-based exercise intervention study in prepubescent and early pubescent children. The Journal of Pediatrics, v. 136, n. 2, p. 156-162, 2000.

KREMER, M. M.; REICHERT, F. F.; HALLAL, P. C. Intensidade e duração dos esforços físicos em aulas de Educação Física. Revista de Saúde Pública, v. 46, n. 2, p. 320-326, 2012.

MOSER, D. C. et al. Pressão arterial elevada, excesso de peso e obesidade abdominal em crianças e adolescentes. Revista da Educação Física, v. 22, n. 4, p. 591-600, 2011.

NADER, P. R. Frequency and intensity of activity of third-grade children in physical education. Archives of Pediatrics \& Adolescent Medicine, v. 157, n. 2, p. 185-190, 2003.

OLIVEIRA, L. et al. Effect of an intervention in Physical Education classes on health related levels of physical fitness in youth. Revista Brasileira de Atividade Física e Saúde, v. 22, n. 1, p. 46-53, 2017.

PEREIRA, F. M. Nível médio de ensino: aulas de educação física como espaço de concretização pedagógica no cotidiano escolar. Revista Pensar a Prática, v. 2, p. 136-155, 1999.

PEREIRA, E. de S. et al. Aptidão física relacionada à saúde em escolares de município de pequeno porte do interior do Brasil. Revista de Educação Física, v. 25, n. 3, p. 459-468, 2014.

RIECK, T. et al. Health-related fitness, body mass index, and risk of depression among adolescents. Medicine and science in sports and exercise, v. 45, n. 6, p. 1083-1088, 2013.

SALLIS, J. F.; MCKENZIE, T. L. Physical education's role in public health. Research Quarterly for Exercise and Sport, v. 62, n. 2, p. 124-137, 1991.

et al. Progress in physical activity over the Olympic quadrennium. The Lancet, v. 388, n. 10051, p. 1325-1336, 2016.

SANTOS, A. L. B. et al. The relationship between pedagogical practices with physical activity levels in classes of Physical Education. Motricidade, v. 13, p. 112-120, 2017. 
STRONG, W. B. et al. Evidence based physical activity for school-age youth. The Journal of Pediatrics, v. 146, n. 6, p. 732-737, 2005.

UNESCO. Diretrizes em educação física de qualidade (EFQ) para gestores de políticas. Brasília, 2015.

WORLD HEALTH ORGANIZATION - WHO. Global recommendations on physical activity for health. 2010.

Recebido em: 07/12/2017

Revisado em: 09/06/2018

Aprovado em: 12/11/2018

Endereço para correspondência:

fernandovian-fw@hotmail.com

Fernando Vian

Universidade Federal do Rio Grande do Sul

Av. Paulo Gama, 110

Farroupilha, 90040-060, Porto Alegre - RS, Brasil 